\title{
Warfarin Therapy and Improved Anticoagulation Control by Patient Self-Management
}

\author{
Leona A. Ritchie ${ }^{1}$ Peter E. Penson ${ }^{1,2}$ Deirdre A. Lane ${ }^{1}$ \\ ${ }^{1}$ Liverpool Centre for Cardiovascular Science, University of Liverpool \\ and Liverpool Heart and Chest Hospital, Liverpool, United Kingdom \\ 2 School of Pharmacy and Biomolecular Sciences, Liverpool John \\ Moores University, Liverpool, United Kingdom \\ Thromb Haemost 2019;119:1550-1552. \\ Address for correspondence Deirdre A. Lane, PhD, Liverpool Centre \\ for Cardiovascular Science, Institute of Ageing and Chronic Disease, \\ University of Liverpool, William Henry Duncan Building, 6 West Derby \\ Street, Liverpool, L7 8TX, United Kingdom \\ (e-mail: deirdre.lane@liverpool.ac.uk).
}

The landscape of oral anticoagulant therapy has significantly changed over the last decade with non-vitamin $\mathrm{K}$ oral anticoagulants (NOACs) now preferred over conventional vitamin $\mathrm{K}$ oral anticoagulants (VKAs), such as warfarin, for a large proportion of patients requiring chronic anticoagulation. ${ }^{1}$ All NOACs have shown a favourable risk/benefit profile compared with warfarin, with significant reductions in intracranial haemorrhage, stroke and systemic embolism. ${ }^{2}$ Their predictable anticoagulant effect without the need for routine monitoring is another attribute which makes them an attractive choice. Nevertheless, warfarin remains a useful drug as many patients requiring OAC have contraindications to NOAC therapy, such as mechanical valve replacement, antiphospholipid syndrome or severely reduced renal function, and globally the NOACs are not always a viable option (either availability to prescribe and/or cost) for many patients.

A recent study by Lutsey et $\mathrm{al}^{3}$ highlights the value of warfarin in an era of NOACs from a patient perspective; a survey on anticoagulant preferences in patients with venous thromboembolism (VTE) found they were most concerned about recurrent VTE and mortality, independent of which anticoagulant they were prescribed. In addition, perceived potential disadvantages of warfarin therapy, such as dietary restrictions and regular monitoring, were generally not considered onerous, whereas the advantages, such as reversibility and the ability to monitor, were viewed favourably. ${ }^{3}$ However, data from the GARFIELD-AF registry suggest that VKA control still remains sub-optimal in clinical practice: mean time in therapeutic range (TTR) was $55 \%$ for 9,934 patients during 1-year follow-up and demonstrated an increased risk of stroke/systemic embolism (hazard ratio [HR] 2.55, 95\% confidence intervals [CIs] 1.61-4.03), major bleeding (HR 1.54, 95\% CI 1.04-2.26) and all-cause mortality (HR 2.39, 95\% CI 1.87-3.06) associated with TTR $<65 \%{ }^{4}$ Consequently, strategies to improve TTR to optimise treatment and prevent adverse outcomes for those patients who

require VKA therapy are needed. The introduction of pointof-care devices such as CoaguChek series, INRatio and Protime allow self-monitoring and self-management and there is an increasing body of evidence to support the implementation of self-management as an alternative to conventional management. However, adherence and persistence with anticoagulation remain treatment challenges.,

In the current issue of Thrombosis \& Haemostasis, Solvik et $\mathrm{al}^{7}$ investigate the effect of switching from conventional warfarin management to self-management on the quality of therapy using five outcome measures: TTR; variance of the international normalised ratio (INR); extreme INR values ( $\leq 1.5$ and $\geq 5$ ); complications (thromboembolic events and major haemorrhage); and patient quality of life (QoL). ${ }^{7}$ They utilised a 'before-and-after' study design whereby 126 patients on warfarin previously conventionally managed, underwent a 21-week training program of warfarin self-management and INRs were recorded for up to 2 years of self-management. Selfmanagement resulted in a significantly higher median TTR (65.9 to $78.1 \%, p<0.001$; Rosendaal method), reduced INR variance $(0.33$ to $0.22, p<0.001)$ and lower percentage of extreme INR values (5.3 to $1.8 \%, p<0.001)$ compared with conventional OAC management. ${ }^{7}$ There were no thromboembolic or major haemorrhage events reported during selfmanagement, compared with $2.4 \%(n=3)$ and $3.2 \%(n=4)$ events, respectively, during conventional management. Selfmanagement also improved patient QoL; there was a significant increase in general management satisfaction and selfefficacy and a reduction in daily hassles, psychological distress and strained social network.

While self-management naturally confers patient autonomy, Solvik et al exclude complete patient autonomy and recommend the on-going involvement of health care practitioners when patients are self-managing, to quickly identify those who are experiencing poor anticoagulation control and to maintain patient motivation. ${ }^{7}$

received

(c) 2019 Georg Thieme Verlag KG Stuttgart · New York
DOI https://doi.org/

10.1055/s-0039-1696982. ISSN 0340-6245. 
This study used multiple measures to assess the quality of warfarin therapy, all of which demonstrate the positive benefit of self-management. The 2-year post-training observation period is also advantageous, as it provides insight into patient willingness to continue with self-management long term, as well as their ability to maintain good INR control. However, the attrition rate raises concerns about the widespread application of self-management strategies; only 77 (61.1\%) patients completed the 2-year self-management period and second QoL assessment. Notably, self-management strategies may only be suitable in a highly selected population. The exclusion of adults $>70$ years old and implementation of a 21-week selfmanagement training program also limits the applicability of the results; the majority of patients who require chronic anticoagulant therapy will be elderly and may be unable to engage with such a lengthy and intensive training program. The present study was not powered to look at hard endpoints like thromboembolism and major bleeding but reassuringly did demonstrate fewer adverse events with self-management.

A 2016 Cochrane review of 28 randomised trials demonstrated that self-management of VKA (for mixed indications) led to a reduction in thromboembolic events (relative risk [RR] 0.47, 95\% CI 0.31-0.70) and all-cause mortality (RR 0.55, 95\% CI 0.36-0.84) but self-management or self-monitoring did not significantly reduce major haemorrhage (RR 0.95 , $95 \% \mathrm{CI}, 0.80-1.12){ }^{8}$ This review also reported mean INR in target range using linear interpolation ( $n=16$ studies) with 15 studies showing improvements with self-management and self-monitoring but only six were statistically significant. ${ }^{8}$ A Danish study comparing patients with VTE who selfmanaged warfarin with a propensity-matched cohort of conventionally managed patients demonstrated a lower rate of recurrent VTE (HR 0.63; 95\% CI 0.42-0.95) and allcause mortality (HR $0.41 ; 95 \% \mathrm{CI} 0.21-0.81$ ) among patients who self-managed but no difference in bleeding (HR 0.95; 95\% CI 0.44-2.02). ${ }^{9}$

The inclusion of QoL as an outcome measure by Solvik et al is noteworthy, as patient-reported outcomes are increasingly important endpoints. The Cochrane review reported 13 trials evaluating QoL with 6 demonstrating better QoL with selfmanagement or self-monitoring, with an improvement in treatment satisfaction. ${ }^{8}$ Patients value improvement in QoL and their viewpoints should be considered when exploring the impact of different management strategies. A recent qualitative study of 40 patient experiences of responsibility for warfarin therapy highlighted that conventional management can cause patients to feel responsible for poor anticoagulation control. This may cause patients to withhold information from anticoagulation care providers and miss appointments, with an overall detrimental effect on clinical outcomes. ${ }^{10}$

The TREAT study highlighted the importance of patient education to improve understanding of the necessity of warfarin and reduce patient perception of treatment harm. Implementation of a one-off, theory-driven educationalbehavioural session significantly improved TTR (Rosendaal method) in atrial fibrillation (AF) patients initiating warfarin during the first 6 months, who's OAC was managed conventionally. ${ }^{11}$ A Cochrane review of educational and behavioural interventions for anticoagulant therapy in patients with AF found small but positive effects of educational interventions on anxiety (mean difference [MD] $-0.62,95 \% \mathrm{CI}-1.21$ to -0.04 ) and depression (MD $-0.74,95 \% \mathrm{CI}-1.34$ to -0.14 ) compared with usual care. ${ }^{12}$

Another important consideration is the cost and costeffectiveness of self-management of VKA; however, Solvik et al do not report on the cost burden of providing a 21-week training program or the cost of testing devices and strips. Cost implications are often a barrier to uptake of selfmanagement strategies, ${ }^{13,14}$ and may be less cost-effective than the current specialised anticoagulation clinics ${ }^{15}$ or in countries, such as Norway, where the quality of conventional OAC management is very good (TTR 70\%). ${ }^{7}$ Arguably, this may reflect upfront training and device costs and selfmanagement costs could reduce over time.

In summary, quality of VKA therapy is likely to improve if self-management is encouraged but the proportion of patients eligible for, and able to perform, self-management long term is limited and cost may be a major barrier to implementation. Even in an era where NOACs may be the preferred alternative, VKA therapy is the mainstay OAC for many patients and strategies to optimise anticoagulation control are paramount. Optimisation of TTR ( $\geq 70 \%)$ is essential to reduce adverse outcomes and other strategies can be utilised, such as patient/carer education and specialised anticoagulant clinics to improve adherence. However, the success of such strategies will likely vary between countries and patient cohorts, depending on the health care system and patient factors, such as education and socio-economic status. Patient values and preferences are crucial, and appropriate engagement encourages patient involvement in their care. ${ }^{16}$

\section{Conflict of Interest}

P.E.P. reports other from AstraZeneca PLC, personal fees from Amgen Inc, other from AKCEA, AMRYT, Link Medical, Napp, and Sanofi, outside the submitted work. D.A.L. reports grants from Boehringer Ingelheim and BristolMyers-Squibb (paid to the institution), and personal fees from Boehringer Ingelheim, Bristol-Myers-Squibb, Bayer, Pfizer, and Daichii-Sankyo outside the submitted work.

\section{References}

1 Lip GYH, Banerjee A, Boriani G, et al. Antithrombotic therapy for atrial fibrillation: CHEST guideline and expert panel report. Chest 2018;154(05):1121-1201

2 Lip G, Freedman B, De Caterina R, Potpara TS. Stroke prevention in atrial fibrillation: past, present and future. Comparing the guidelines and practical decision-making. Thromb Haemost 2017;117 (07):1230-1239

3 Lutsey PL, Horvath KJ, Fullam L, et al. Anticoagulant preferences and concerns among venous thromboembolism patients. Thromb Haemost 2018;118(03):553-561

4 Haas S, Ten Cate H, Accetta G, et al; GARFIELD-AF Investigators. Quality of vitamin k antagonist control and 1-year outcomes in patients with atrial fibrillation: a global perspective from the GARFIELD-AF Registry. PLoS One 2016;11(10):e0164076

5 Hylek EM. Treatment persistence in atrial fibrillation: the next major hurdle. Thromb Haemost 2018;118(12):2018-2019 
6 Raparelli V, Proietti M, Cangemi R, Lip GY, Lane DA, Basili S. Adherence to oral anticoagulant therapy in patients with atrial fibrillation. Focus on non-vitamin $\mathrm{K}$ antagonist oral anticoagulants. Thromb Haemost 2017;117(02):209-218

7 Solvik ULE, Kristofferson AH, Brodin E, Averina M, Sandberg S. Quality of warfarin therapy and quality of life are improved by self-management for two years. Thromb Haemost 2019;119(09): $1632-1641$

8 Heneghan CJ, Garcia-Alamino JM, Spencer EA, et al. Self-monitoring and self-management of oral anticoagulation. Cochrane Database Syst Rev 2016;7:CD003839

9 Larsen TB, Skjøth F, Grove EL, Nielsen PB, Christensen TD. Effectiveness of self-managed oral anticoagulant therapy in patients with recurrent venous thromboembolism. A propensity-matched cohort study. Thromb Haemost 2016;116(03):524-529

10 Gillespie C, Rose AJ, Petrakis BA, Jones EA, Park A, McCullough MB. Qualitative study of patient experiences of responsibility in warfarin therapy. Am J Health Syst Pharm 2018;75(22):1798-1804

11 Clarkesmith DE, Pattison HM, Lip GY, Lane DA. Educational intervention improves anticoagulation control in atrial fibrilla- tion patients: the TREAT randomised trial. PLoS One 2013;8(09): e74037

12 Clarkesmith DE, Pattison HM, Khaing PH, Lane DA. Educational and behavioural interventions for anticoagulant therapy in patients with atrial fibrillation. Cochrane Database Syst Rev 2017;4:CD008600

13 Jennings I, Kitchen D, Keeling D, Fitzmaurice D, Heneghan C; BCSH Committee. Patient self-testing and self-management of oral anticoagulation with vitamin $\mathrm{K}$ antagonists: guidance from the British Committee for Standards in Haematology. Br J Haematol 2014;167(05):600-607

14 Jowett S, Bryan S, Murray E, et al. Patient self-management of anticoagulation therapy: a trial-based cost-effectiveness analysis. Br J Haematol 2006;134(06):632-639

15 Connock M, Stevens C, Fry-Smith A, et al. Clinical effectiveness and cost-effectiveness of different models of managing long-term oral anticoagulation therapy: a systematic review and economic modelling. Health Technol Assess 2007;11(38):iii-iv

16 Loewen PS, Ji AT, Kapanen A, McClean A. Patient values and preferences for antithrombotic therapy in atrial fibrillation. A narrative systematic review. Thromb Haemost 2017;117(06):1007-1022 